\title{
Practice of High-density 3D Seismic Exploration in Tight Oil Exploration in G Area of Central Sichuan
}

\author{
Tan Rongbiao, Wang Xiaolan, Deng Xiaojiang, Huang Lijuan, Li Yangjing, Zhang Ruyue
}

Southwest Geophysical Institude of China National Petroleum Corporation, Chengdu, China

\author{
Email address: \\ tanrongbiao@cnpc.com.cn (Tan Rongbiao)
}

\section{To cite this article:}

Tan Rongbiao, Wang Xiaolan, Deng Xiaojiang, Huang Lijuan, Li Yangjing, Zhang Ruyue. Practice of High-density 3D Seismic Exploration in Tight Oil Exploration in G Area of Central Sichuan. American Journal of Mechanics and Applications. Vol. 9, No. 1, 2021, pp. 1-5. doi: 10.11648/j.ajma.20210901.11

Received: November 22, 2020; Accepted: December 24, 2020; Published: January 22, 2021

\begin{abstract}
The Oil reservoir in Jurassic of Sichuan Basin is tight, thin, high heterogeneity, complex sand distribution and well-developed micro-fault etc., whose difference of buried depth is up to $2000 \mathrm{~m}$ from south to north. The vertical and lateral resolution of conventional seismic exploration is too low to predict reservoir clearly, which restrains further development of close oil exploitation in central of Sichuan Basin. Lots of examples shows that high density acquisition is able to improve the description ability of seismic data, In G area of Sichuan Basin, a high-density, high-fold, small-cell, full-azimuthal 3D acquisition has been tested, which is the first mountainous high-density seismic exploration in China. In view of mass data and difficult geological target, a processing technique series including auto-first-break-picking, pre-stack noise suppression, robust surface consistent deconvolution, pre-stack time migration in OVT (Offset Vector Tile) field is applied to obtain high quality image. Analysis of multiple geological target results revealed that, the high-density data has fair higher signal-to-noise ratio, much wider bandwidth with more information, much better vertical and lateral resolution, which increased the identification of sand boundary, predication of thin reservoir, discrimination of micro-fault and fracture, recognition of sub geological features. It is well provided that high-density 3D seismic exploration has vast application future in close oil exploitation.
\end{abstract}

Keywords: High-density Three-dimensional, Fully Automatic First-time Picking,

Robust Surface Consistency Deconvolution Technology, OVT Domain Processing, Application Effect

\section{Overview}

Many years of exploration of tight oil layers in the Gongshanmiao area have shown that the area is dominated by thin interbeds, with a single layer thickness of $2-10 \mathrm{~m}$, an average porosity of less than $2 \%$, and a permeability of less than $0.1 \mathrm{mD}$. It is an ultra-low porosity and ultra-low permeability reservoir. The reservoir has strong spatial heterogeneity and well developed fractures. As tight oil layers enter the development stage, seismic data need to be used to predict the thickness of thin reservoirs and the spatial distribution of small fractures. This poses a challenge to the vertical and horizontal resolution of seismic data. Therefore, high-density 3D seismic tests have been carried out in this area. Through high-density seismic exploration, the distribution characteristics of channel reservoir and fracture development characteristics in this area are proved, which provides support for the deployment of development wells $[5]$.

\section{High-density 3D Seismic Acquisition}

The pilot test uses Western Qiko's UniQ integrated single-point land seismic acquisition system, which can use high-density, shallow well and small-charge explosive sources, with up to 45,000 single-point accelerating detectors, providing seismic sampling up to 1 millisecond. data. Considering the geological characteristics of the area, the design of the observing system needs to ensure adequate and intensive sampling requirements, as well as adequate protection of the high and low frequency components of the effective information and the accuracy of high spatial sampling. The main construction parameters are as follows [6]. 


\subsection{Observing System Parameters [7]}

Observing system: 36 lines $\times 20$ guns $\times 720$ channels orthogonal.

Panel size: $5 \mathrm{~m} \times 5 \mathrm{~m}$ Cover times: $18 \times 18$.

Distance: $10 \mathrm{~m}$ gun interval: $10 \mathrm{~m}$.

Receiving line spacing: 200m gun line Distance: 200m.

Maximum longitudinal distance: $3595 \mathrm{~m}$ Maximum non-positive distance: $3595 \mathrm{~m}$.

Maximum offset: $5085 \mathrm{~m}$ Aspect ratio: 1.

\subsection{Excited and Received Parameters}

Excitation mode: excitation in the well.

Well Depth: Production of wells with a depth of $\geq 6 \mathrm{~m}$ and a single deep well with a micro-measurement well depth of $\geq 15 \mathrm{~m}$.

Dosage: $1 \mathrm{~kg}$ of mudstone; $1.5 \mathrm{~kg}$ of sandstone.

\section{High-density 3D Data Processing}

In view of the geological characteristics of tight oil exploration and the characteristics of high-density collection, the focus of this treatment is on seismic data fidelity and increasing vertical and horizontal resolution [1]. The huge problem in processing is how to perform static correction, denoising, amplitude preservation and pre-stack time migration on the high-density collected massive data. Here, four characteristic technologies are used to complete the processing efficiently and with high quality.

\subsection{Fully Automatic First Arrival Picking Technology}

The automatic picking technology fully considers the surface factors, acquisition factors and environmental factors to complete the fast and accurate picking of the first arrival. The basic technical process of picking: data preprocessing -> batch processing automatic processing $\rightarrow$ attribute-based editing $->$ quality control space single shot picking pass rate. Statistical analysis of the first-time pick-up results of the whole work area shows that the average pick-up rate in the whole work area can reach $86 \%$ [4].

\subsection{Prestack Denoising}

Due to different surface excitation conditions, large differences in source energy, and various types of excitation background noise, the amplitude energy difference between the gun and the gun and between the road and the road is large. In order to make the noise and signal more regular, especially to minimize the influence of the amplitude variation on the signal without harming the effective signal, preliminary surface consistency amplitude compensation processing is needed before denoising to avoid energy. The unbalanced negative effect on the denoising process, the amplitude recovery is performed after the denoising is completed, and the fine amplitude processing is performed again on the denoised data [2].

After abnormal energy attenuation $\rightarrow>$ surface wave suppression -> scattering surface wave suppression -> environmental noise attenuation, the single-shot signal-to-noise ratio is greatly improved, and the energy is more balanced. In the above process, adhering to the principle of "removing the noise and not hurting the signal", some residual noise still remains on the single gun, which can be completely removed by subsequent CMP domain denoising, data regularization and offset processing.

\subsection{Robust Surface Consistency Deconvolution}

The robust surface consistency deconvolution technique is a new generation of surface consistency algorithm that combines surface consistency amplitude processing with deconvolution processing. This algorithm can better resist the influence of strong noise on the solution result by solving the equation of robust L1/L2 norm when solving the equation. The narrowing of the seismic wavelet, the weakening of the wavelet transform, the weakening of the high-frequency noise, and the widening of the effective frequency band of the signal are beneficial to improve the vertical resolution of the data [3].

\subsection{OVT Domain Processing Technology}

OVT technology is a data processing domain developed in recent years for wide-azimuth (omnidirectional) seismic acquisition data.

The size of the OVT is determined by the gun line spacing and the detection line spacing. The number of OVTs is equal to the number of coverages. Each OVT has a limited range of offset and azimuth. If the corresponding OVTs of all the cross-aligned track sets are extracted, the OVT gathers will be a single coverage data body covering the entire work area, so it can be used independently for the offset, so that the azimuth and offset information can be saved after the offset. For azimuth analysis, this is the most advantageous aspect of OVT technology. However, this single-coverage, like the conventional co-offset, also encounters problems caused by irregularities in the observing system, such as voids. The diagonal OVT merge (that is, the diagonal vector slices whose azimuth angles are 180 degrees apart) can complement each other to illuminate the holes, and form a data subset of the two overlays, so that the impact of the offset after the offset is minimized. The average offset and azimuth of each OVT gather are calculated separately as the representative offset and azimuth of the gather. This method is superior to the fixed offset range and the azimuth separation data method.

\section{Data Analysis}

\subsection{High-density 3D Seismic Imaging}

Compared with the conventional three-dimensional ratio, the thin layer of the target layer in the region, the large-scale development of crack clusters of different scales and the spatial extension of the channel sand, the high-density three-dimensional earthquake has more detailed descriptions, rich information, and the characteristics of the wave group on the section are clear and small. The size of the river is clearly 
visible.

It can be seen from the conventional three-dimensional and high-density three-dimensional offset profiles of the Well1 well (Figure 1) that the high-density three-dimensional signal-to-noise ratio is higher, the vertical and lateral resolutions are higher, the fault breakpoints are more clearly, and the imaging accuracy is improved. Greatly improved. In the comparison of high-density three-dimensional and conventional three-dimensional offset time slices (Figure 2), it can be seen that the large fault surface of the fault is clear and clear, and the fault contact relationship is clear. In particular, the VTI prestack time migration is more clear on the fracture surface of the north-south large fault, the breakpoint homing is more accurate, the contact relationship is more clear; the spatial distribution of the multi-group crack system is more detailed; the channel sand body The identification of spatial development features and the prediction of thickness and boundaries are more helpful.
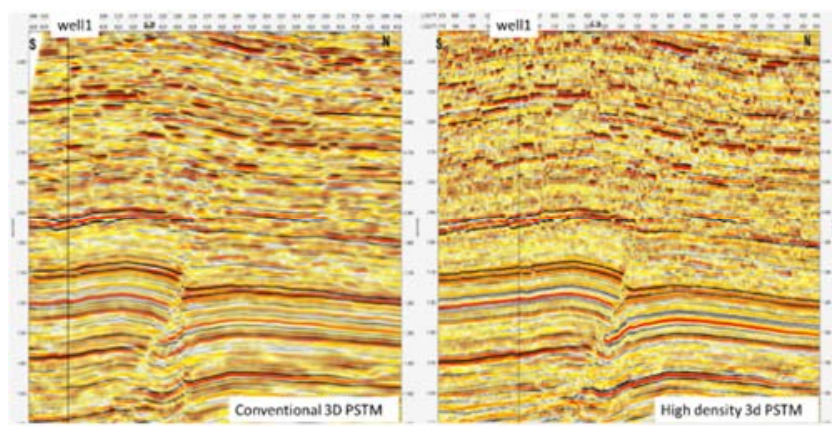

Figure 1. Comparison of conventional three-dimensional (left) and high-density three-dimensional (right) offset profiles in Well1 well.

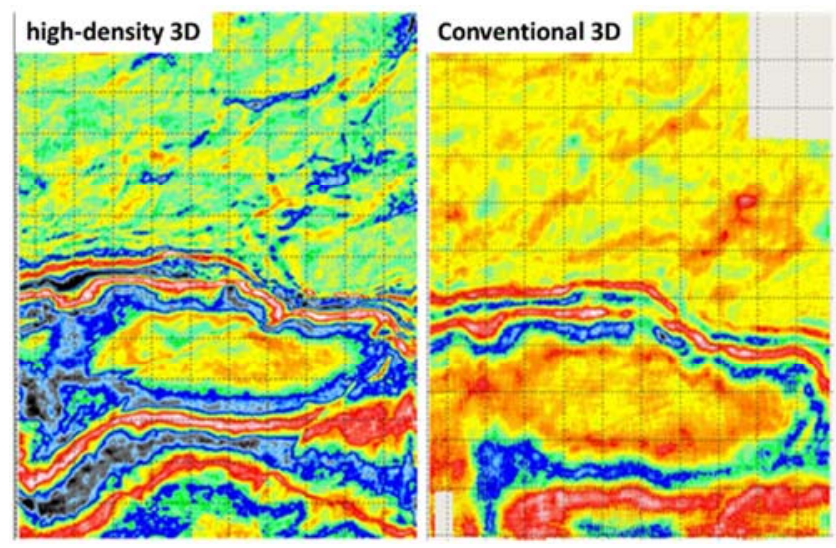

Figure 2. Conventional (right) and high-density three-dimensional (left) pre-stack offset horizontal slices.

\subsection{Geological Effect}

From the perspective of data quality, the signal-to-noise ratio and resolution of the 3D seismic section are high, and the characteristics of the reflected wave group are obvious. The diffraction wave and the rotating wave are reasonably homing, the fold characteristics are clear, the section is clear, and the structure can be reasonably and reliably reflected. The relationship between the target layer and the jurassic axis is better, the resolution is higher, the bandwidth is wider than usual, and the low frequency and high frequency information is more abundant. Figure 3 is a comparison of the conventional and high-density cross-sections of the Well2 well. The vertical resolution of the high-density data is higher. The target layer bandwidth is around $8-115 \mathrm{~Hz}$ and the main frequency is $55 \mathrm{~Hz}$. The geological phenomenon is clear. The characterization of thin fractures and the resolution of thin layers are more precise and reliable. On the conventional data, the in-phase axis wrinkles or deflection caused by small or micro-fractures, the high-density data is clearly disconnected from the same phase axis, and the breakpoints are more clearly characterized and can be distinguished. $6 \mathrm{~m}$ break distance fault.

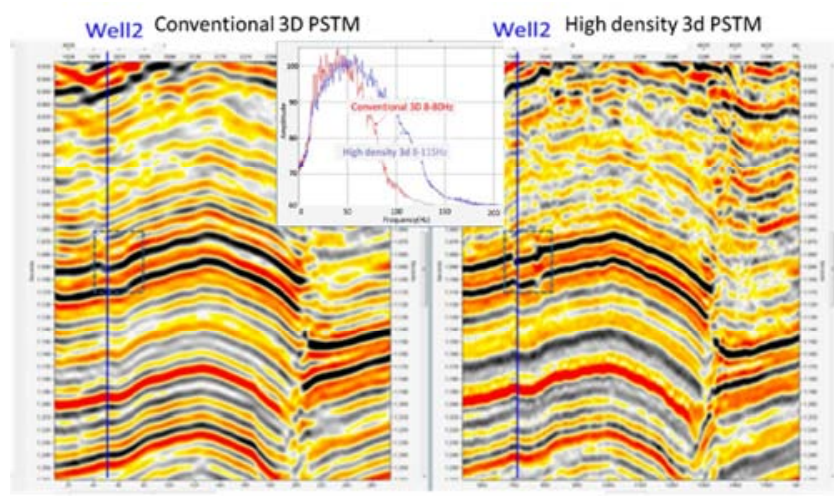

Figure 3. Conventional (left) and high density (right) offset profiles and spectra over the Well2 well.

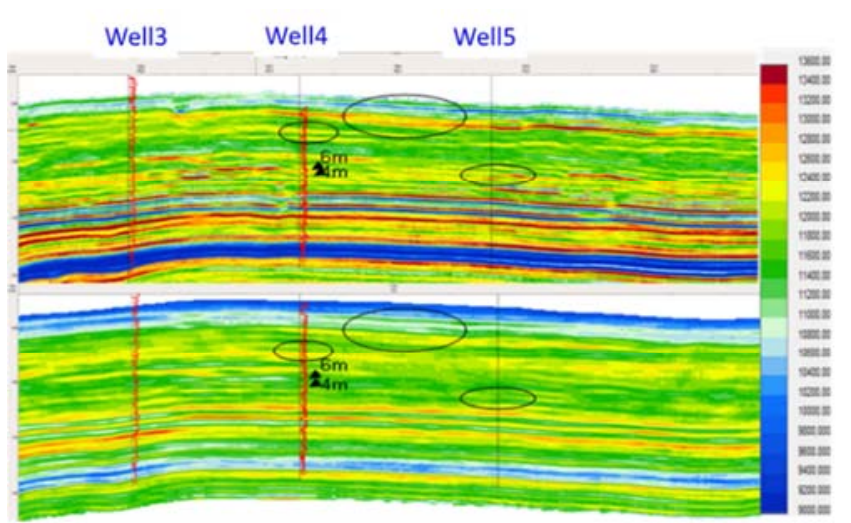

Figure 4. Well inversion profile of well Well 3-4-5.

The above one is the result of high-density data, the next one is the result of conventional data, and the insertion curve is the GR curve.

The lateral resolution is higher, and the lateral change boundary of the lithology is more clearly characterized. In the river sand body characterization of Shaximiao Formation (see Figure 5), the conventional data is not clear due to the lateral sampling rate, and the river boundary is relatively fuzzy. The Well6 well in the early stage of the sand channel of the Shahejie River is more accurate because of the conventional data. Poor, not designed to enter the target, the target front displacement increased by 170.7 meters compared with the design; in addition, the small river channel or the reticulated river with a river width less than $0.2 \mathrm{~km}$ could not be clearly 
reflected.
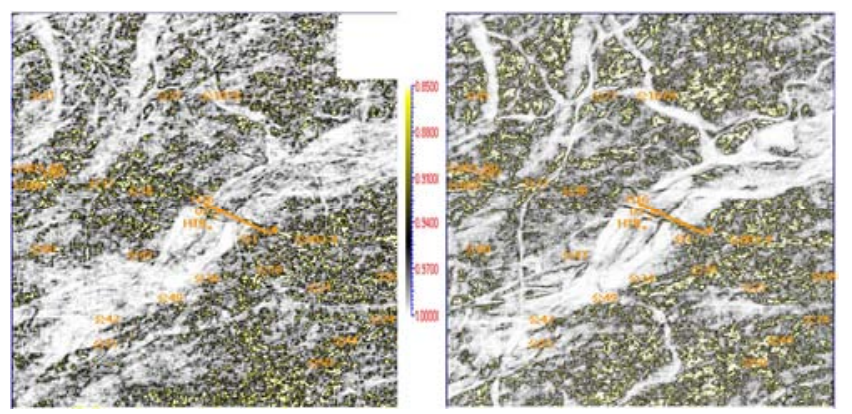

Figure 5. Comparison of the effects of the middle and lower rivers in the Sha section of Shaximiao Formation.

Up to $45 \mathrm{~ms}$ similarity slice on the bottom of the sand (conventional 3D-left) $45 \mathrm{~ms}$ similarity slice on the bottom of the sand (high-density 3D-right)

The lateral change of the physical properties of the Da'anzhai thin limestone is clear. Figure 6 is the velocity inversion profile along the Well1 well trajectory. It can be clearly seen that in the horizontal trajectory section, the thickness of the junior limestone is stable, and the speed of the conventional three-dimensional inversion is very weak, indicating that the physical properties are basically unchanged, high. Density reservoir inversion has higher vertical and horizontal resolution, and the velocity variation inside limestone is more clearly described.

The prediction of the micro-fracture zone and the fracture development zone is more clear.

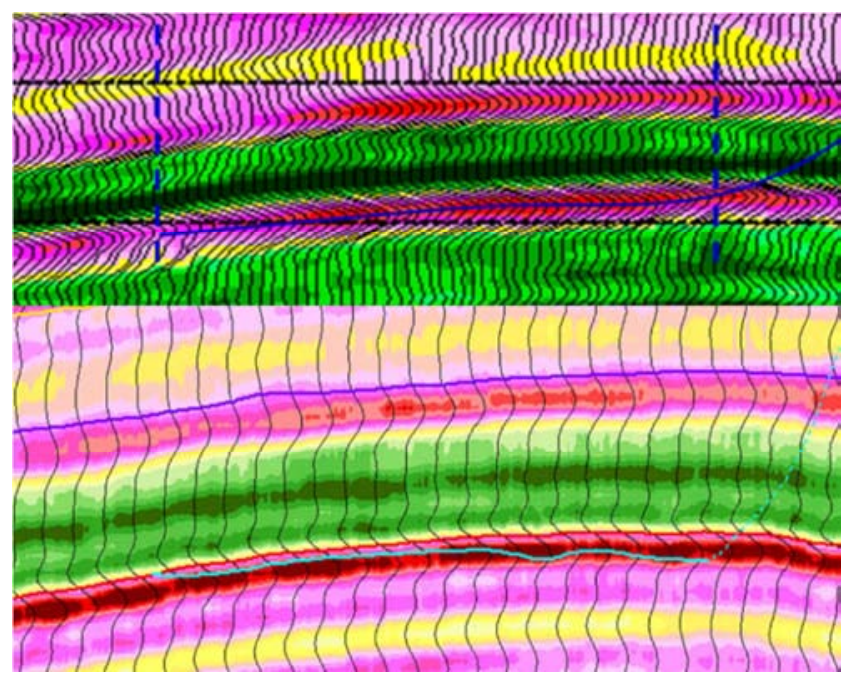

Figure 6. Speed inversion profile section of Well7 well drilling trajectory.

The above is the result of regular data, the next one is the result of high-density data.

Due to the insufficient lateral sampling rate, the conventional data cannot be identified for the fracture zone width smaller than the CDP dot-distance micro-fracture zone. In the demonstration and design of the $115 \mathrm{H}$ well, the crack response characteristics are not seen according to the conventional 3D seismic data, but on the high-density 3D seismic data, it is obvious that the obvious network crack zone develops along the well track direction. Figure 7 is a high-density prediction of the distribution of fracture network and microseismic monitoring results in the Dasan sub-section of Da'anzhai. The distribution of cracks in the first, fifth, sixth, seventh, eighth, and tenth stages of microseismic monitoring is consistent with the predicted fracture development, and the crack patterns at all levels in the microseismic monitoring results are There is a certain difference, indicating that the reservoir stress is more complex and the natural fractures develop, proving that the natural fracture network is existing. The results of fracturing microseismic monitoring from Well7 show that the fracture zone characterized by high-density 3D seismic is reliable.

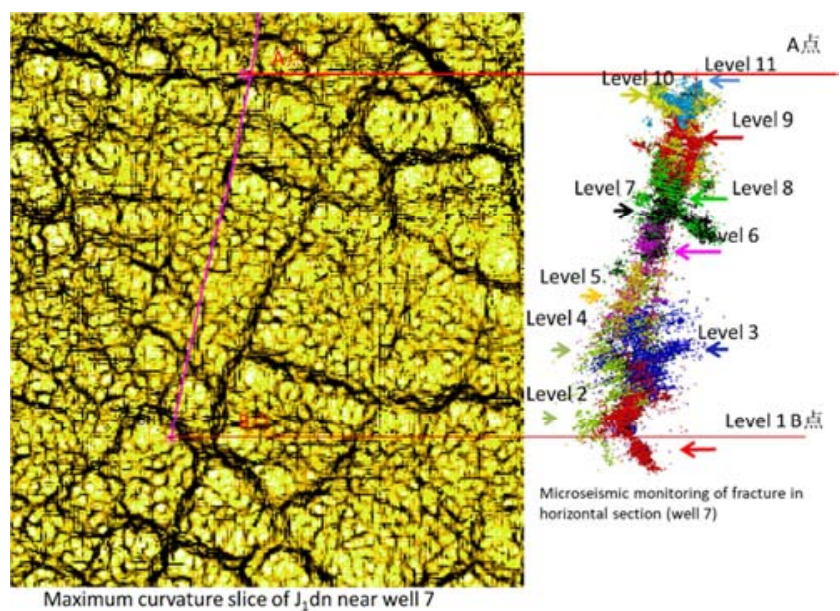

Figure 7. Comparison of prediction results between fracture development zone and microseismic monitoring in Well7 well.

\section{Conclusion}

High-density 3D seismic exploration in Area $G$ is China's first high-density acquisition project for mountain seismic exploration, and has obtained high-quality seismic data with rich reflection information. Through the implementation of the project, a mountain high-density acquisition processing interpretation exploration technology was initially formed.

Through the preliminary application of high-density three-dimensional in the G-zone, the effective frequency band of seismic data has been significantly broadened, laying a solid foundation for thin reservoir and crack prediction, which has improved the sand body resolution to $4 \mathrm{~m}$ and the fault resolution to $6 \mathrm{~m}$. The spatial resolution of the micro-fracture zone and the fracture zone is improved, which greatly satisfies the "three high" high signal-to-noise ratio, high-resolution, high-fidelity earthquake in the unconventional ultra-low porosity and low permeability tight reservoir of the Middle Jurassic [8]. The demand for data provides a new idea for the exploration and development of tight oil in Chuanzhong. It is believed that with the further maturity and deepening of high-density seismic exploration technology and the deepening of application practice, high-density 3D seismic exploration and development of dense oil and gas in Sichuan It has broad application prospects [9]. 


\section{References}

[1] Zhang Yonggang, Wang Wei, Yin Junjie, etc., Single Point High Density Seismic Data Processing Analysis and Preliminary Evaluation, Petroleum Geophysical Exploration, 2010, 45 (2).

[2] Duan Wensheng, Li Fei, Wang Yanchun, Wang Chunhe, Dang Qingning et al., Anti-collision vector chip technology for wide-azimuth seismic processing, Petroleum Geophysical Prospecting, 2013, 48 (2).

[3] Liu Xinxin, Wu Guozhen, Liang Wei, et al. A review of single-point high-density seismic exploration techniques, Progress in Geophysics, 2009, 24 (4).

[4] Li Xinxiang, 3D seismic total offset vector path gather, Petroleum Geophysical Exploration, 2007, 46 (6).

[5] Gao Qiuju, Han Wengong, Chen Xiangpeng, etc., Luojia high-density 3D seismic data interpretation and effect, SPG/SEG Shenzhen 2011 International Geophysical Conference Proceedings.

[6] Liu Erpeng, Research on High-density Seismic Acquisition Technology, Taiyuan University of Technology, Master thesis, 2011.

[7] Wang Naijian, Application Research of High-density Seismic Acquisition Technology in Tabei Region, China University of Petroleum, Master thesis, 2008.

[8] Liu Zhenwu, Saliming, Dong Shitai, Tang Donglei, Practice and Future of China Petroleum High-Density Earthquake Technology, 2009, 36 (2).

[9] Cai Xiyuan, Han Wengong, Yu Jing, Shi Linguang, High-density 3D seismic exploration example in Luojia area, Petroleum Geophysical Prospecting, 2011, 46 (2). 